\title{
Sobrecarga de Trabalho dos Profissionais de Enfermagem: fatores de interface a Síndrome de Burnout
}

\author{
Work overload of Nursing professionals: interface factors to Burnout Syndrome \\ Sobrecarga de trabajo de los profesionales de Enfermería: factores de interfaz al Síndrome de
}

Burnout

Recebido: 07/02/2022 | Revisado: 14/02/2022 | Aceito: 17/02/2022 | Publicado: 26/02/2022

\author{
Shirley Moreira Cintra \\ ORCID: https://orcid.org/0000-0001-8167-6966 \\ Unidade de Ensino Superior de Feira de Santana, Brasil \\ E-mail: 7shirleymoreiracintra@gmail.com \\ Bárbara Angélica Santos de Oliveira \\ ORCID: https://orcid.org/0000-0003-4585-3560 \\ Universidade Estadual de Feira de Santana, Brasil \\ E-mail: barbaradioliveira@gmail.com \\ Lucas Souza Almeida de Araujo \\ ORCID: https://orcid.org/0000-0001-5723-6725 \\ Universidade Estadual de Feira de Santana, Brasil \\ E-mail: lucas.araujx@hotmail.com \\ Juan Victor de Almeida dos Santos \\ ORCID: https://orcid.org/0000-0002-7052-9570 \\ Universidade do Estado da Bahia, Brasil \\ E-mail: contato.jvas@gmail.com \\ Maria Emília Cirqueira Silva \\ ORCID: https://orcid.org/0000-0002-8556-482X \\ Faculdade Anísio Teixeira, Brasil \\ E-mail: emiliacirqueira@gmail.com \\ Gustavo Marques Porto Cardoso \\ ORCID: https://orcid.org/0000-0002-0125-6492 \\ Secretaria de Educação do Estado da Bahia, Brasil \\ E-mail: gugampc@hotmail.com
}

\begin{abstract}
Resumo
Objetivo: identificar na literatura quais fatores influenciam na sobrecarga de trabalho dos profissionais de enfermagem e sua interface com a Síndrome de Burnout. Metodologia: trata-se de uma revisão integrativa de abordagem qualitativa e caráter descritivo, com recorte temporal de 2012 a 2021, a base de dados eletrônica selecionada para busca foi a BVS por contemplar as bases SCIELO e LILACS. Os artigos foram selecionados primeiramente pela leitura do título, posteriormente pelos resumos e por último, leitura completa. Resultados: evidenciou-se o total de nove publicações que conceberam os critérios da pesquisa, pois os mesmos contemplam constructo teórico para esta revisão. Considerações finais: possibilitou a identificação dos fatores contribuintes para a síndrome de burnout e a necessidade de realizar pesquisas acerca de reflexões a respeito das possíveis estratégias para o enfrentamento da síndrome. Se faz necessário maiores investimentos em estudos sobre Síndrome de Burnout, pois estratégias de intervenções devem ser elucidadas para serem aplicadas numa urgência ou emergência na Saúde Pública, que permitam a prevenção, tratamento adequado e intervenção quando necessário, favorecendo a diminuição de profissionais acometidos pela síndrome.
\end{abstract}

Palavras-chave: Enfermeiro; Burnout; Saúde do trabalhador; Enfermagem.

\begin{abstract}
Objective: to identify in the literature which factors influence the workload of nursing professionals and its interface with the Burnout Syndrome. Methodology: this is na integrative review with a qualitative approach and descriptive character, with a time frame from 2012 to 2021, the electronic database selected for Search was BVS because it includes the bases SCIELO and LILACS. The articles weew selected first by Reading the title, later by the abstracts and finally, full reading. Results: there was a total of nine publications that designed the research criteria, as They include a theoretical constructo for this review. Final considerations: it enabled the identification of contributing factors for the burnout syndrome and the need to conduct research on reflections on possible strategies for coping with the syndrome. Greater investments are needed in studies on Burnout Syndrome, as intervention strategies must be elucidated to be applied in an urgency or emergency in Public Health, allowing prevention, adequate treatment and intervention when necessary, favoring the reduction of professionals affected by the syndrome.
\end{abstract}

Keywords: Nurse; Burnout; Occupational health; Nursing. 


\section{Resumen}

Objetivo: identificar en la literatura qué factores influyen en la sobrecarga de trabajo de los profesionales de enfermería y su interfaz con el Síndrome de Burnout. Metodología: se trata de una revisión integradora con enfoque cualitativo y carácter descriptivo, con un marco temporal de 2012 a 2021, la base de datos electrónica seleccionada para la búsqueda fue la BVS por incluir las bases de datos SCIELO y LILACS. Los artículos fueron seleccionados primero por la lectura del título, luego por los resúmenes y finalmente, por la lectura completa. Resultados: se evidenciaron un total de nueve publicaciones que concibieron los criterios de investigación, ya que incluyen un constructo teórico para esta revisión. Consideraciones finales: permitió la identificación de factores contribuyentes al síndrome de burnout y la necesidad de realizar investigaciones sobre reflexiones sobre posibles estrategias de enfrentamiento del síndrome. Se necesitan mayores inversiones en estudios sobre el Síndrome de Burnout, ya que se deben dilucidar estrategias de intervención para ser aplicadas en una urgencia o emergencia en Salud Pública, que permitan la prevención, el tratamiento adecuado y la intervención cuando sea necesario, favoreciendo la reducción de profesionales afectados por el síndrome.

Palabras clave: Enfermera; Agotamiento; Salud del trabajador; Enfermería.

\section{Introdução}

O contentamento no ambiente de trabalho em saúde é um fenômeno complexo, podendo ser influenciado por múltiplos fatores. Um baixo nível de satisfação dos profissionais que trabalham no Sistema Único de Saúde (SUS) pode afetar o relacionamento com os usuários e interferir no serviço oferecido, dentre estes profissionais, o de enfermagem.

Segundo Oliveira et al. (2019) o grau de insatisfação do enfermeiro pode prejudicar a harmonia e estabilidade dentro do local de trabalho, consequentemente, a satisfação do paciente, pois intrinsicamente está relacionada ao cuidado prestado pelo profissional, indicando que uma percepção negativa do usuário é reflexo de uma assistência não efetiva dentro do sistema.

Os profissionais de enfermagem são submetidos a diversos fatores que ocasionam sobrecarga de trabalho. O impacto sobre a saúde e a vida destes trabalhadores relaciona-se com a percepção de alta demanda nas situações rotineiras e dificuldades no enfrentamento diante das exigências profissionais estabelecidas (Alves et al., 2018). A insatisfação com o trabalho pode desencadear a diminuição da realização pessoal e redução da autoestima.

A sobrecarga de trabalho é definida como a exaustão do trabalhador, atingindo uma sensação de estar no limite de uma somatória de situações estressantes, tendo como resultado o cansaço e a falta de paciência (Fernandes, Medeiros, Ribeiro, 2008). Estas cargas de trabalho causam impactos no corpo do trabalhador, gerando desgaste e adoecimento. Quando há uma grande exaustão este trabalhador pode estar sendo acometido por alguma síndrome, sendo a mais comum à de Burnout (Mota, 2020).

Nesse contexto, a Síndrome de Burnout (SB) ou Síndrome do Esgotamento Profissional foi descrita pela primeira vez em 1974, e desde então vem sendo estudada por vários pesquisadores (Moreira et al., 2009). Este distúrbio pode ser desenvolvido pela exposição exagerada a estressores emocionais e interpessoais, ocorrendo com mais frequência em trabalhadores que precisem se relacionar com o público. A enfermagem é identificada como uma das profissões de maior incidência de SB (Paiva et al., 2019).

Essa síndrome é determinada por três fatores: exaustão emocional, despersonalização e baixa realização profissional. Sendo reconhecida mundialmente e considerada um grande problema psicossocial na saúde dos trabalhadores (Silva et al., 2015).

Sendo assim, justifica-se este estudo pela relevância social e científica da identificação dos fatores de interface a Síndrome de Burnout nestes trabalhadores. Portanto, conhecer o contexto no qual essa assistência se desenvolve e seu impacto no mundo do trabalho possibilita uma reflexão acerca das reais necessidades dos profissionais, criando a possibilidade de minimizar os impactos negativos relacionados a sobrecarga de trabalho, insatisfação e esgotamento emocional, promovendo alterações na qualidade da assistência.

Destarte, este estudo tem como objetivo identificar na literatura já publicada quais fatores influenciam na sobrecarga de trabalho dos profissionais de enfermagem e sua interface com a Síndrome de Burnout. 


\section{Metodologia}

Trata-se de um artigo de revisão integrativa de literatura sobre os fatores que influenciam na sobrecarga de trabalho dos profissionais de enfermagem desencadeando a Síndrome de Burnout. Que abrange pesquisas realizadas anteriormente, que possibilitam obter conclusões ao assunto de interesse. Foram incluídos artigos entre 2012 a 2021, indexados na base de dados da Biblioteca Nacional de Saúde (BVS), independente do periódico de publicação.

A revisão integrativa permite uma abordagem metodológica extensa ao admitir revisões de estudos experimentais e não experimentais, teóricos e empíricos, pois pela sua essência qualitativa propõe compreender o tema analisado de forma ampla (Souza et al., 2010).

Para a estratégia de busca inicialmente foi verificado se as palavras-chaves escolhidas estavam cadastradas nos Descritores em Ciências da Saúde (DECs), a saber: Enfermeiro e Burnout, os quais foram combinados pelo operador booleano AND.

Foram adotados os critérios de inclusão: resumo disponível nas bases de dados, artigos publicados na integra e em língua portuguesa, disponíveis online e gratuitos, ter sido publicado entre 2012 a 2021, que sua abordagem tenha a temática proposta. Utilizaram-se as seguintes bases de dados: Literatura Latino Americana e do Caribe em Ciências e Saúde (LILACS), Base de Dados de Enfermagem (BDENF), Sistema Online de Busca e Análise de Literatura Médica (MEDLINE), Scientific Electronic Library Online (SCIELO). Sendo os critérios de exclusão: não estar em formato de artigo, estar fora do período de publicação, fora da temática e em idiomas não nativos.

Como protocolo de tratamento dos dados optou-se pela análise de conteúdo temática, empregado por Minayo (2012) definidas da seguinte maneira: fase exploratória da pesquisa, que se inicia ainda no projeto de investigação com a escolha do material a formulação dos objetivos da análise. O segundo momento que ocorrera a dedicação coleta de dados onde se reuni as informações que irão responder à pergunta da investigação. E por último o tratamento do material encontrado que se subdivide em três fases.

A primeira delas é a ordenação, onde será realizado o mapeamento coletado e sua organização, em seguida será processada à classificação dos dados a partir dos questionamentos que direcionam a pesquisa, identificando o que surge de relevante para a investigação: pôr fim a análise propriamente dita, onde será executada a articulação a articulação dos dados e o que já se tem de produção cientifica relacionada ao conteúdo, respondendo as inquietações da pesquisa que serão traçadas a partir do objetivo do estudo (Minayo, 2012).

\section{Resultados}

Foram encontrados 231 artigos, os quais tiveram todos os títulos lidos, tendo sido excluídos 215 artigos. Foram realizados leitura dos resumos dos 16 restantes, sendo excluídos 07 . Os 09 artigos que restaram foram lidos na íntegra e utilizado no presente estudo, pois os mesmos contemplam constructo teórico para esta revisão (Figura 1). 
Figura 1 - Processo metodológicos da revisão, 2021.
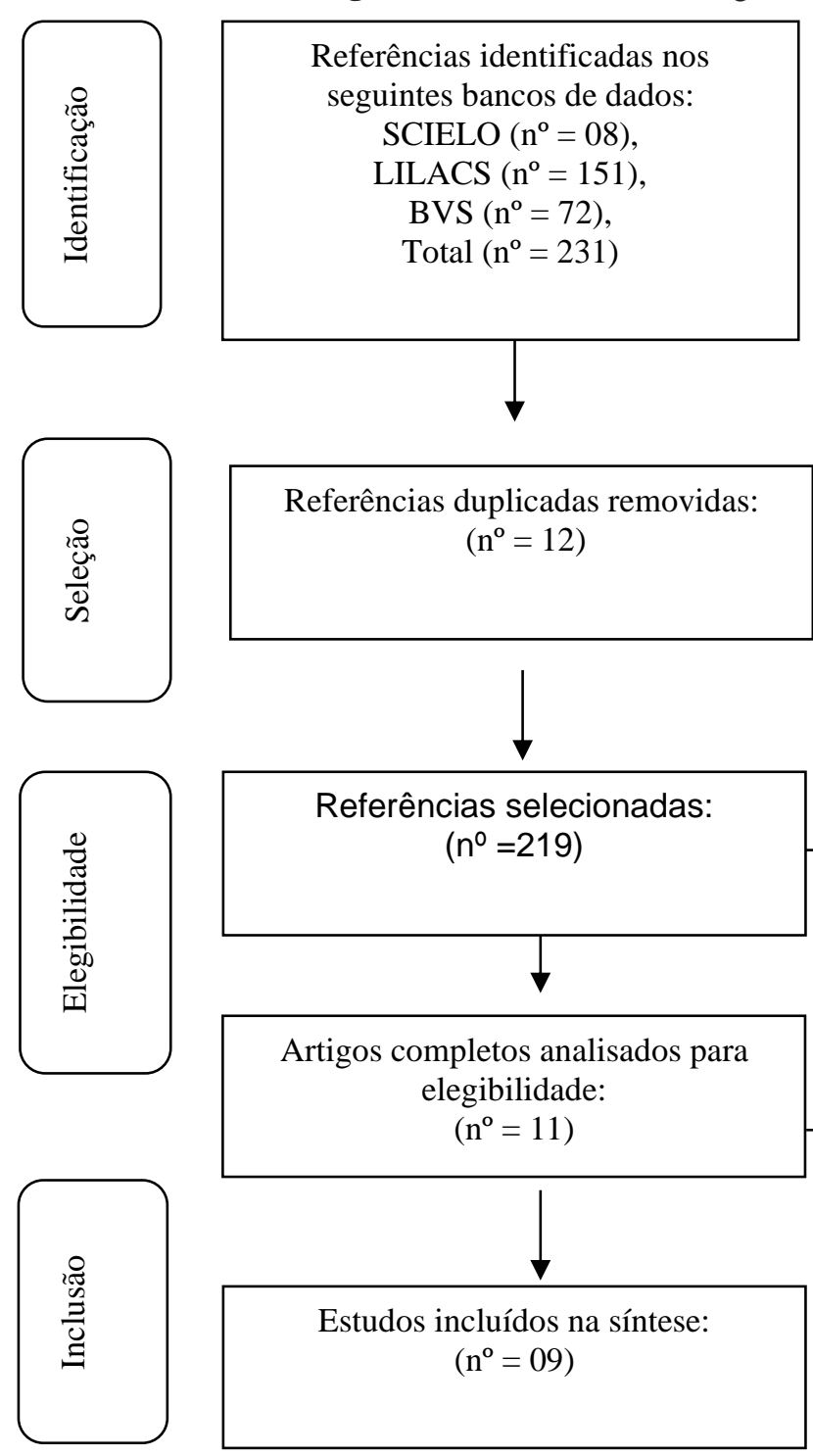

Referências excluídas por título e resumo: $\left(n^{\circ}=208\right)$

Artigos completos excluídos da análise: $\left(\mathrm{n}^{\circ}=02\right)$

Fonte: Próprios autores (2021).

O corpus documental deste estudo foi constituído por 09 artigos científicos que foram publicados no período de 2013 a 2021, 03 artigos publicados em 2018, os outros 06 artigos foram publicados um único artigo nos anos 2014, 2015, 2016, 2017, 2019 e 2021. Em relação aos periódicos, foram publicados em Revista Latino-Americana de Enfermagem, Revista Brasileira de Ciências da Saúde, Revista de Enfermagem UFPE Online, Revista Baiana de Saúde Pública, CuidArte Enfermagem, Psicologia Reflexão e Crítica e PSICO (PUCRS. ONLINE) (Quadro 1). 
Quadro 1 - Corpus documental da revisão integrativa, 2021.

\begin{tabular}{|c|c|c|c|c|c|c|}
\hline ID & AUTOR/ANO & TÍTULO & $\begin{array}{l}\text { PERIÓDICO DE } \\
\text { PUBLICAÇÃO }\end{array}$ & QUALIS & MÉTODO & $\begin{array}{c}\text { FATORES CONTRIBUINTES PARA SÍNDROME DE } \\
\text { BURNOUT }\end{array}$ \\
\hline 01 & $\begin{array}{l}\text { BORGES et al., } \\
2021 .\end{array}$ & $\begin{array}{l}\text { Burnout entre enfermeiros: um estudo multicêntrico } \\
\text { comparativo }\end{array}$ & $\begin{array}{l}\text { Revista Latino- } \\
\text { Americana de } \\
\text { Enfermagem. }\end{array}$ & A1 & Coorte Transversal & $\begin{array}{ll} & \text { Realização Profissional; } \\
\text { - } & \text { Despersonalização; } \\
\text { - } \quad \text { Baixa realização pessoal; } & \text { Exaustão emocional. } \\
\end{array}$ \\
\hline 02 & RAMOS et al., 2019. & $\begin{array}{l}\text { Impactos da Síndrome de Burnout na qualidade de vida } \\
\text { dos profissionais de enfermagem da atenção básica a } \\
\text { saúde }\end{array}$ & $\begin{array}{l}\text { Revista Brasileira de } \\
\text { Ciências da Saúde }\end{array}$ & B2 & Coorte Transversal & $\begin{array}{ll} & \text { Relações conflituosas dentro da equipe; } \\
\text { - } & \text { Nível de qualidade de vida; } \\
\text { - } & \text { Exaustão emocional; } \\
\text { Despersonalização. }\end{array}$ \\
\hline 03 & $\begin{array}{l}\text { SILVA; FARIAS, } \\
2018 .\end{array}$ & Qualidade de Vida e estresse dos enfermeiros. & $\begin{array}{l}\text { Revista de } \\
\text { Enfermagem UFPE } \\
\text { Online. }\end{array}$ & B2 & $\begin{array}{l}\text { Revisão Integrativa de } \\
\text { Literatura }\end{array}$ & $\begin{array}{ll} & \text { Estresse } \\
\text { - } & \text { Condições de insalubridade no ambiente de trabalho; } \\
\text { - } & \text { Sobrecarga de trabalho; } \\
\text { - } \quad \text { Longa jornada de trabalho; } \\
\text { - } \quad \text { Besvalorização da profissão; } \\
\text { - } \quad \text { Turnos inflexíníveis. } \\
\end{array}$ \\
\hline 04 & $\begin{array}{l}\text { VIDOTTI et al., } \\
2018 .\end{array}$ & $\begin{array}{l}\text { Síndrome de Burnout e o trabalho em turnos na equipe } \\
\text { de enfermagem. }\end{array}$ & $\begin{array}{l}\text { Revista Latino- } \\
\text { Americana de } \\
\text { Enfermagem. }\end{array}$ & $\mathrm{A} 1$ & Estudo transversal & $\begin{array}{ll} & \text { Exaustão emocional; } \\
\text { - } & \text { Alta despersonalização; } \\
\text { - } & \text { Baixa realização profissional; } \\
\text { - } & \text { Estresse ocupacional; } \\
\text { - } & \text { Baixo apoio social; } \\
\text { - } & \text { Incivilidade laboral; } \\
\end{array}$ \\
\hline 05 & $\begin{array}{l}\text { DORIGAN; } \\
\text { GUIRARDELLO, } \\
2018 .\end{array}$ & $\begin{array}{l}\text { Efeito do ambiente da prática do enfermeiro nos } \\
\text { resultados do trabalho e clima de segurança. }\end{array}$ & $\begin{array}{l}\text { Revista Latino- } \\
\text { Americana de } \\
\text { Enfermagem. }\end{array}$ & A1 & $\begin{array}{l}\text { Estudo quantitativo do } \\
\text { tipo correlacional }\end{array}$ & $\begin{array}{ll} & \text { Exaustão emocional; } \\
\text { - } & \text { Sobrecarga; } \\
\text { Esgotamento dos recursos físicos e emocionais. }\end{array}$ \\
\hline 06 & $\begin{array}{l}\text { MOURÃO et al., } \\
2017 .\end{array}$ & Síndrome de Burnout no contexto da enfermagem. & $\begin{array}{l}\text { Revista Baiana de } \\
\text { Saúde Pública. }\end{array}$ & B3 & $\begin{array}{l}\text { Revisão narrativa de } \\
\text { Literatura }\end{array}$ & $\begin{array}{l}\text { - Insatisfação no ambiente de trabalho; } \\
\text { - } \quad \text { Estresse; } \\
\text { - Condições laborais difíceis. }\end{array}$ \\
\hline 07 & $\begin{array}{l}\text { SANCHEZ; } \\
\text { OLIVEIRA, } 2016 .\end{array}$ & $\begin{array}{l}\text { Aspectos mediadores e desencadeadores da Síndrome de } \\
\text { Burnout nos enfermeiros. }\end{array}$ & $\begin{array}{l}\text { CuidArte } \\
\text { Enfermagem. }\end{array}$ & B2 & $\begin{array}{l}\text { Revisão Integrativa de } \\
\text { Literatura }\end{array}$ & $\begin{array}{ll} & \text { Desgaste dos trabalhadores; } \\
\text { - } & \text { Exaustão emocional; } \\
\text { - } & \text { Sobrecarga laboral; } \\
\text { - } & \text { Falta de reconhecimento; } \\
\text { - } \quad \text { Conflitos interpessoais. }\end{array}$ \\
\hline 08 & $\begin{array}{l}\text { CAMPOS et al., } \\
2015 .\end{array}$ & $\begin{array}{l}\text { Fatores Sociodemográficos e Ocupacionais Associados à } \\
\text { Síndrome de Burnout em Profissionais de Enfermagem. }\end{array}$ & $\begin{array}{l}\text { Psicologia Reflexão e } \\
\text { Crítica }\end{array}$ & B1 & Coorte Transversal & $\begin{array}{ll}\text { - } & \text { Exaustão emocional; } \\
\text { - } & \text { Insatisfação laboral. }\end{array}$ \\
\hline 09 & $\begin{array}{l}\text { NEVES; } \\
\text { OLIVEIRA; } \\
\text { ALVES, } 2014 .\end{array}$ & $\begin{array}{l}\text { Síndrome de Burnout: impacto da satisfação no trabalho } \\
\text { e da percepção de suporte organizacional. }\end{array}$ & $\begin{array}{l}\text { PSICO (PUCRS. } \\
\text { ONLINE). }\end{array}$ & B3 & Coorte Transversal & 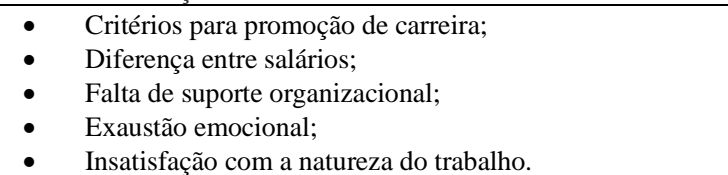 \\
\hline
\end{tabular}

Fonte: Próprios autores (2021). 


\section{Discussão}

Os fatores de interface a Síndrome de Burnout identificados no corpus documental foram: Realização profissional, Despersonalização, Baixa realização pessoal, Exaustão emocional, Relações conflituosas dentro da equipe, Nível de qualidade de vida, Estresse e condições de insalubridade no ambiente de trabalho, Sobrecarga de trabalho, Longa jornada de trabalho, Desvalorização profissional, Baixos salários, Turnos inflexíveis, Baixo apoio social, Incivilidade laboral, Insatisfação financeira, Exaustão emocional, Esgotamento de recursos físicos e emocionais, Condições laborais difíceis, Falta de reconhecimento, Baixo nível de suporte, Conflitos interpessoais, Insatisfação laboral, Critérios para promoção de carreira, Diferença entre salários e Falta de suporte organizacional (Quadro 1).

A baixa realização profissional, pessoal e insatisfação no ambiente de trabalho reflete uma autoavaliação negativa do desempenho, desmotivação e sensação de inadequação pessoal, evidenciando um declínio do sentimento de competência e produtividade, causando prejuízos no local laboral (Borges et al., 2021; Vidotti et al., 2018; Mourão et al., 2017; Campos et al., 2015; Neves; Oliveira; Alves, 2014). É necessário estratégias e ações para propiciar melhorias efetivas na vida do profissional.

A despersonalização é explicitada pelos pesquisadores Borges et al. (2021) e Vidotti et al. (2018) por se caracterizar como insensibilidade emocional, atitudes e pensamentos negativos, sendo identificada pela perda de significado do trabalho e distanciamento com a equipe.

Um fator em comum evidenciado por muitos autores, diz respeito à exaustão emocional, que consiste em uma das primeiras manifestações da Síndrome de Burnout, por se referir ao sentimento de sobrecarga dos profissionais. A exaustão é descrita pelo esgotamento, cansaço, desgaste, esvaziamento afetivo, falta de energia e entusiasmo (Borges et al., 2021; Silva; Farias, 2018; Vidotti et al., 2018; Dorigan; Guirardello, 2018; Sanchez; Oliveira, 2016; Campos et al., 2015; Neves; Oliveira; Alves, 2014). Desse modo, é relevante frisar a necessidade de medidas para a redução dos fatores predisponentes, para prevenir e reduzir síndromes relacionadas ao trabalho.

Outra questão que os pesquisadores Ramos et al. (2019); Sanchez e Oliveira (2016) trazem é as relações conflituosas dentro da equipe de enfermagem, por ser um fator predisponente para o desenvolvimento da Síndrome, é imprescindível valorizar o trabalho em equipe. A incivilidade laboral é exteriorizada por atitudes que infringem as normas sociais de conduta cortês e harmoniosa (Vidotti et al., 2018). A qualidade de vida destes profissionais possui forte ligação com o êxito das necessidades pessoais, por esta razão, o ambiente deve conceder sensação de satisfação e conforto para os enfermeiros.

O estresse no âmbito do trabalho excede os níveis adaptativos e se cronifica, afetando o grau de satisfação pessoal e profissional, produzindo consequências negativas na perspectiva individual, familiar e social. Esse fator vivenciado pelos profissionais de enfermagem prejudica seu desempenho no atendimento ao público (Silva; Farias, 2018; Vidotti et al., 2018; Mourão et al., 2017).

Em decorrência da longa jornada de trabalho, turnos inflexíveis e falta de suporte organizacional, os profissionais de enfermagem se submetem a desgastes físicos e emocionais, levando esse profissional a uma vida estressante (Silva; Farias, 2018; Sanchez; Oliveira, 2016; Neves; Oliveira; Alves, 2014). Destaca-se a necessidade de gestores de recursos humanos buscarem meios para recuperar o equilíbrio entre as expectativas e realidade desses profissionais.

As condições laborais difíceis e de insalubridade no ambiente de trabalho afetam substancialmente a saúde dos profissionais de enfermagem, podendo desencadear afecções ocupacionais como a Síndrome de Burnout (Silva; Farias, 2018; Mourão et al., 2017). Assim sendo, é essencial a detecção precoce dos fatores determinantes para o risco da síndrome, sendo necessário intervenções no momento oportuno.

A desvalorização da enfermagem através dos baixos salários ocasiona uma insatisfação financeira, esse desagrado levaos ao desgaste, limitações e ao adoecimento. A enfermagem é uma classe com baixos salários e que não é remunerada de acordo com a qualificação, competência, capacidade profissional e funções que desempenha, causando uma adversidade com a 
promoção de carreira da profissão. Essa falta de reconhecimento desencadeia fatores para desenvolvimento da Síndrome de Burnout. (Silva \& Farias, 2018; Vidotti et al., 2018; Sanchez \& Oliveira, 2016; Neves et al., 2014).

De acordo com Vidotti et al. (2018) o baixo apoio social é um fator desencadeador da Síndrome de Burnout, sendo necessário para evitar esse agente ter um ambiente de trabalho amistoso e com apoio mútuo de supervisores.

Este estudo apresentou algumas limitações que se referem aos próprios critérios de inclusão, como seleção de artigos apenas no idioma português, a busca de artigos em apenas uma base de dados eletrônica e a forma de combinação dos descritores. Mesmo assim, por se tratar de uma temática de interesse pela comunidade científica apresenta relevância social, cientifica e técnica para exploração de dados.

\section{Considerações Finais}

O desenvolvimento do presente estudo possibilitou a identificação dos fatores contribuintes para a Síndrome de Burnout, além de elevar o conhecimento sobre a doença ocupacional, que apesar de muitas pesquisas e publicações sobre o tema, apenas uma quantidade reduzida realiza reflexões a respeito das possíveis estratégias para o enfretamento da síndrome.

Ao realizar a pesquisa foi identificado que os enfermeiros manifestam os sinais e sintomas característicos da Síndrome de Burnout ou pelo menos estão sob o risco de desenvolvê-la, comprometendo a qualidade de vida desses profissionais.

Se faz necessário maiores investimentos em estudos sobre Síndrome de Burnout, pois estratégias de intervenções devem ser elucidadas para serem aplicadas numa urgência ou emergência na Saúde Pública, que permitam a prevenção, tratamento adequado e intervenção quando necessário, favorecendo a diminuição de profissionais acometidos pela síndrome.

Ressalta-se que, aliado a isso, os gestores tomem conhecimento que a prevenção de fatores precursores da síndrome trará benefícios para toda instituição que o profissional atua. Essas estratégias permitirão o alcance de maior qualidade dos serviços prestados, beneficiando o indivíduo, as organizações de saúde e a sociedade.

No que se refere ao objetivo principal, este foi alcançado. Espera-se, assim, que este estudo contribua para o desenvolvimento de novas pesquisas nessa área, acerca das variáveis aqui discutidas, provocando possíveis reconfigurações e reformulações da realidade inerente a essa profissão, com o objetivo de ampliar o conhecimento referente a Sobrecarga de Trabalho dos Profissionais de Enfermagem: Fatores de Interface a Síndrome de Burnout.

\section{Referências}

Alves, S. R. (2018). et al. Serviços de saúde mental: percepção da enfermagem em relação à sobrecarga e condições de trabalho. Revista Online de Pesquisa, 10(1), 25-29. https://doi.org/10.9789/2175-5361.2018.v10i1.25-29.

Borges, E. M. das N. (2021). et al. Burnout entre enfermeiros: um estudo multicêntrico comparativo. Revista Latino-Americana De Enfermagem, 29(3432), 0111, out. https://doi.org/10.1590/1518-8345.4320.3432.

Brasil. (2012). Ministério da Saúde. Conselho Nacional de Saúde. Comissão Nacional de Ética em Pesquisa. Resolução $n^{\circ} 466$, de 12 de dezembro de 2012. Aprova as diretrizes e normas regulamentadoras de pesquisas envolvendo seres humanos. http://bvsms.saude.gov.br/bvs/saudelegis/cns/2013/res0466_12_12_2012.html.

Campos, I. C. M. (2015). et al. Fatores Sociodemográficos e Ocupacionais Associados à Síndrome de Burnout em Profissionais de Enfermagem. Psicologia Reflexão e Crítica, 28(4), 764-771. https://doi.org/10.1590/1678-7153.201528414.

Dorigan, G. H. \& Guirardello, E. B. (2018). Efeito do ambiente da prática do enfermeiro nos resultados do trabalho e clima de segurança. Revista LatinoAmericana de Enfermagem, 26, 01-08. https://doi.org/10.1590/1518-8345.2633.3056.

Fernandes, S. M. B. A., Medeiros, S. M. \& Ribeiro, L. M. (2008). Estresse ocupacional e o mundo atual: repercussões na vida cotidiana das enfermeiras. Revista Eletrônica de Enfermagem, 10(2), 414-427. https://doi.org/10.5216/ree.v10i2.8043.

Minayo, M. C de S. (2012). Análise qualitativa: teoria, passos e fidedignidade. Ciência \& saúde coletiva, 17, 621-626. https://www.scielo.br/j/csc/a/39YW8sMQhNzG5NmpGBtNMFf/?lang=pt\&format=pdf.

Moreira, D. S. (2009). et al. Prevalência da Síndrome de Burnout em trabalhadores de enfermagem de um hospital de grande porte da Região Sul do Brasil. Caderno de Saúde Pública, 25(7), 1559-1568. https://www.scielo.br/j/csp/a/pc7N3MpyPZGTkWLVXYtWhKN/?format=pdf\&lang=pt. 
Research, Society and Development, v. 11, n. 3, e36411326699, 2022

(CC BY 4.0) | ISSN 2525-3409 | DOI: http://dx.doi.org/10.33448/rsd-v11i3.26699

Moreno, F.N. (2011). et al. Estratégias e intervenções no enfrentamento da síndrome de burnout. Revista Enfermagem, 19(1). 140-145. C:IUsers\AlonsolDocuments\1 r (bauru.sp.gov.br).

Mota, B. S. (2020). et al. As contribuições da síndrome de burnout para o déficit do trabalho da enfermagem: revisão integrativa da literatura. Revista Eletrônica Acervo Saúde, 12(10), e4383-e4383. https://doi.org/10.25248/reas.e4383.2020.

Mourão, A. L. (2017). et al. Síndrome de Burnout no contexto da enfermagem. Revista Brasileira de Saúde Pública, 41(1), 131-143. https://doi.org/10.22278/2318-2660.2017.v41.n1.a1926.

Neves, V. F., Oliveira, Á.de F. \& Alves, P. C. (2014). Síndrome de Burnout: Impacto da Satisfação no Trabalho e da Percepção de Suporte Organizacional. Psico, 45(1), 45-54. https://dialnet.unirioja.es/servlet/articulo?codigo=5633364.

Oliveira, J. F. (2019). et al. Satisfação profissional e sobrecarga de trabalho de enfermeiros da área de saúde mental. Ciência \& Saúde Coletiva. 24(7), 25932598. https://doi.org/10.1590/1413-81232018247.20252017.

Paiva, J. D. M. (2019). et al. Fatores desencadeantes da Síndrome de Burnout em enfermeiros. Revista de Enfermagem. 13(2), 483-190. https://doi.org/10.5205/1981-8963-v13i02a235894p483-490-2019.

Ramos, C. E. B. (2019). et al. Impactos da Síndrome de Burnout na qualidade de vida dos profissionais de enfermagem da atenção básica a saúde. Revista Brasileira de Ciências da Saúde. 23(3), 285-296. https://doi.org/10.22478/ufpb.2317-6032.2019v23n3.43595.

Sanchez, F. F. S. \& Oliveira, R. (2016). Aspectos mediadores e desencadeadores da Síndrome de Burnout nos enfermeiros. CuidArte Enfermagem. 10(1), 6167. http://fundacaopadrealbino.org.br/facfipa/ner/pdf/CuidarteEnfermagemvolume10Jan-Jun2016.pdf.

Santos, K. C. R. (2020). Síndrome de Burnout: Definição, fatores causadores e possibilidades de enfrentamento. Revista Científica Multidisciplinar Núcleo do Conhecimento. 09(2), 12-20. 32749/nucleodoconhecimento.com.br/saude/possibilidades-de-enfrentamento.

Silva, G. K. \& Farias, S. N. P. (2018). Qualidade de Vida e estresse dos enfermeiros. Revista de Enfermagem UFPE Online. 12(12), 3378-3385. https://doi.org/10.5205/1981-8963-v12i12a236158p3378-3385-2018.

Silva, S. C. P. S. (2015). et al. A Síndrome de Burnout em profissionais da Rede de Atenção Primária à Saúde de Aracaju, Brasil. Ciência \& Saúde Coletiva. 20(10), 3011-3020. https://doi.org/10.1590/1413-812320152010.19912014.

Vidotti, V. (2018). et al. Síndrome de Burnout e o trabalho em turnos na equipe de enfermagem. Revista Latino-Americana De Enfermagem. 26(55), 355-365. https://doi.org/10.4025/arqmudi.v24i1.50051. 